\title{
Disk-mediated accretion burst in a high-mass young stellar object
}

\author{
A. Caratti o Garatti ${ }^{1 \star}$, B. Stecklum ${ }^{2}$, R. Garcia Lopez ${ }^{1}$, J. Eislöffel ${ }^{2}$, T. P. Ray ${ }^{1}$, A. Sanna ${ }^{3}$, R. Cesaroni ${ }^{4}$, \\ C. M. Walmsley ${ }^{1,4}$, R. D. Oudmaijer ${ }^{5}$, W. J. de Wit ${ }^{6}$, L. Moscadelli ${ }^{4}$, J. Greiner ${ }^{7}$, A. Krabbe ${ }^{8}$, C. Fischer $^{8}$, \\ R. Klein ${ }^{9}$ and J. M. lbañez ${ }^{10}$
}

Solar-mass stars form via disk-mediated accretion. Recent findings indicate that this process is probably episodic in the form of accretion bursts ${ }^{1}$, possibly caused by disk fragmentation $^{2-4}$. Although it cannot be ruled out that high-mass young stellar objects arise from the coalescence of their low-mass brethren ${ }^{5}$, the latest results suggest that they more likely form via disks ${ }^{6-9}$. It follows that disk-mediated accretion bursts should occur ${ }^{10,11}$. Here we report on the discovery of the first disk-mediated accretion burst from a roughly twenty-solar-mass high-mass young stellar object ${ }^{12}$. Our near-infrared images show the brightening of the central source and its outflow cavities. Near-infrared spectroscopy reveals emission lines typical for accretion bursts in low-mass protostars, but orders of magnitude more luminous. Moreover, the released energy and the inferred mass-accretion rate are also orders of magnitude larger. Our results identify disk-accretion as the common mechanism of star formation across the entire stellar mass spectrum.

S255IR NIRS 3 (aka S255IR-SMA1) is a well-studied $\sim 20 \mathrm{M}_{\odot}$ $\left(\mathrm{L}_{\mathrm{bol}} \sim 2.4 \times 10^{4} \mathrm{~L}_{\odot}\right)$ high-mass young stellar object $(\mathrm{HMYSO})^{13,14}$ in the S255IR massive star-forming region ${ }^{13}$, located at a distance of $\sim 1.8 \mathrm{kpc}^{15}$. It exhibits a disk-like rotating structure ${ }^{13}$, very likely an accretion disk, viewed nearly edge-on ${ }^{16}$ (inclination angle $\sim 80^{\circ}$ ). A molecular outflow has been detected ${ }^{13}$ (blueshifted lobe position angle (P.A.) $\sim 247^{\circ}$ ) perpendicular to the disk. Two bipolar lobes (cavities), cleared by the outflow, are illuminated by the central source and show up as reflection nebulae towards the southwest (blueshifted lobe) and northeast (redshifted lobe, see Fig. 1, left panel). At $\sim 2$ '.5 west of NIRS 3, another HMYSO, NIRS 1 (aka S255IR-SMA2; $\mathrm{M}_{*} \sim 8 \mathrm{M}_{\odot}$; ref. 14), is also seen in the near infrared.

Following the detection of a $6.7 \mathrm{GHz}$ class II methanol maser flare in the S255IR star-forming region ${ }^{17}$, we performed near-infrared imaging with the Panoramic Near Infrared Camera (PANIC) at the Calar Alto Observatory in November 2015 (see Methods), to check whether the flare was triggered by an accretion burst from one of the massive protostars in the region ${ }^{12}$. Indeed, infrared radiation from heated dust emitting at $\sim 20-30 \mu \mathrm{m}$ is thought to be the pumping mechanism of this maser transition ${ }^{18}$.

Our images in the $H(1.65 \mu \mathrm{m})$ and $\mathrm{Ks}(2.16 \mu \mathrm{m})$ bands reveal an increase in the infrared brightness (burst) of S255IR NIRS 3, by $\Delta H \sim 3.5 \mathrm{mag}$ and $\Delta K \sim 2.5 \mathrm{mag}$ with respect to the latest archival images taken with the UKIRT Infrared Deep Sky Survey (UKIDSS) in December 2009 (see Fig. 1, upper left and upper right panels). Moreover, a substantial increase in brightness is also observed in the bipolar outflow cavities, which scatter the light from the central accreting source. These findings provide evidence of an accretion burst onto the HMYSO. The lower left panel of Fig. 1 shows the brightness ratio between the first PANIC Ks-band image and the UKIDSS K-band frame (see Methods). The relative brightness distribution exhibits a bipolar appearance. In principle, this effect could be the result of enhanced scattering in the outflow lobes or extinction variability. However, the former would require an increase in the number density of grains by an order of magnitude, which is impossible to obtain within the short time between the UKIDSS and PANIC images. Extinction variability is also excluded by our multi-wavelength observations, which include near-, mid- and far-infrared spectroscopy and imaging (see Methods). Therefore, the only explanation for this phenomenon is that we are observing the light from the burst scattered by the dust in the outflow cavities (the so-called light echo). Indeed, subsequent PANIC imaging confirms this hypothesis by verifying the motion of the light echo, between November 2015 and February 2016 (see Fig. 1, lower right panel), as it moves away from the source. This discovery allows us to approximately date the onset of the burst to around mid-June 2015 (see Methods). Remarkably, this is the first light echo ever observed from the outburst of a high-mass young star.

The SINFONI/VLT K-band spectrum of NIRS 3 (see Fig. 2, left panel, black spectrum), obtained on the 26th of February 2016, shows a very red and almost featureless continuum, much brighter than that observed with the same instrument in the quiescent phase in $2007^{14}$ (see Fig. 2, left panel, red spectrum). Notably, no photospheric features in absorption are detected. The lack of prominent features and the extremely reddened continuum are probably due to: the strong veiling, caused by the accretion; the high visual extinction ( $A_{V}=44 \pm 16 \mathrm{mag}$, see Methods), resulting from the large inclination of the circumstellar disk to our line of sight; and the presence of a thick envelope surrounding the HMYSO.

In contrast, K-band integral field spectroscopy of the redshifted lobe (the brightest outflow cavity, see Fig. 1), performed with SINFONI/VLT (March 2016) and NIFS/Gemini (April 2016), reveals a wealth of spectral features from the burst (see Fig. 2, right

\footnotetext{
${ }^{1}$ Dublin Institute for Advanced Studies, Astronomy \& Astrophysics Section, 31 Fitzwilliam Place, Dublin 2, Ireland. ${ }^{2}$ Thüringer Landessternwarte Tautenburg, Sternwarte 5, D-07778 Tautenburg, Germany. ${ }^{3}$ Max Planck Institut für Radioastronomie, Auf dem Hügel 69, D-53121 Bonn, Germany. ${ }^{4}$ INAF-Osservatorio Astrofisico di Arcetri, Largo E. Fermi 5, 50125 Firenze, Italy. ${ }^{5}$ School of Physics and Astronomy, University of Leeds, Leeds LS2 9JT, UK. ${ }^{6}$ ESO-European Organisation for Astronomical Research in the Southern Hemisphere, Alonso de Cordova 3107, Vitacura, Santiago de Chile, Chile. ${ }^{7}$ Max-Planck Institut für Extraterrestrische Physik, D-85741 Garching, Germany. ${ }^{8}$ Deutsches SOFIA Institut, Pfaffenwaldring 29, D-70569 Stuttgart, Germany. ${ }^{9}$ NASA Ames Research Center, Moffett Field, California 94035, USA. ${ }^{10}$ Instituto de Astrofísica de Andalucía (CSIC), Glorieta de la Astronomía 3, E-18008 Granada, Spain. *e-mail: alessio@cp.dias.ie
} 

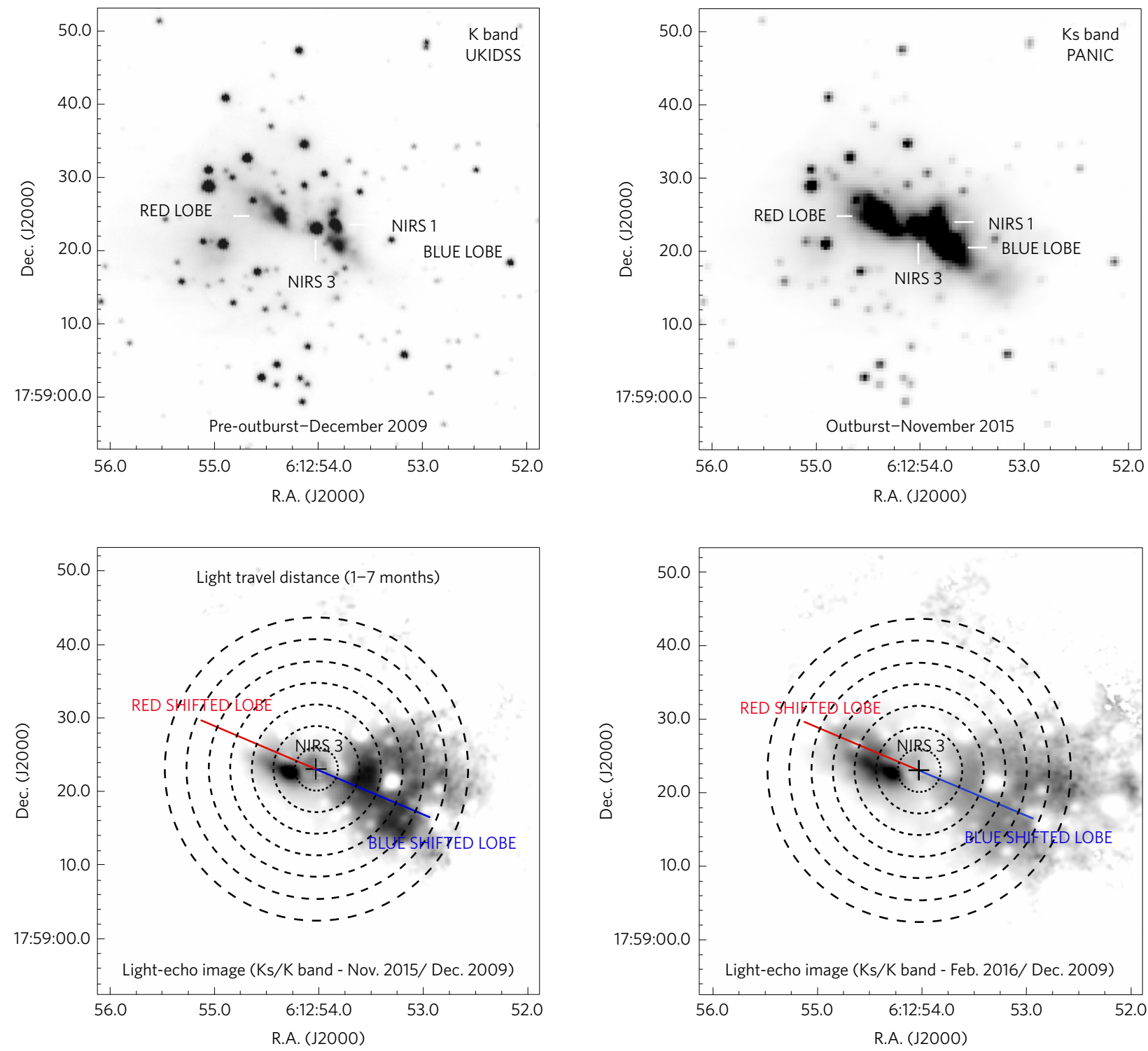

2.4

$3.8 \quad 5.2$

6.6

8

9.4

11

12

14

Figure 1 | Pre-outburst, outburst and brightness-ratio images of S255IR NIRS 3. Upper left: UKIDSS pre-outburst K-band image, December 2009: NIRS 3 is centred on a bipolar nebula, towards northeast and southwest, namely the red- and blueshifted outflow cavities of the protostar. Another HMYSO (NIRS 1) is situated $\sim 2.5^{\prime \prime}$ west of NIRS 3. Upper right: PANIC outburst Ks-band image (November 2015), showing the brightening of NIRS 3 and its outflow cavities. Lower left: Ratio between PANIC Ks (November 2015) and UKIDSS K (December 2009) images. The gradual increment of brightness ratio towards the HMYSO represents the light echo-a record of the burst history. The echo asymmetry is primarily due to the outflow inclination with respect to the sky plane. For guidance concentric circles mark light travel distances in the plane of the sky separated by one month. Lower right: Ratio between PANIC Ks (February 2016) and UKIDSS K (December 2009) images showing the motion of the light echo. The lower bar indicates the range of the relative brightness increase.

panel, black spectrum). Indeed, the visual extinction towards the lobes $\left(\mathrm{A}_{V} \sim 18 \pm 5 \mathrm{mag}\right.$ and $28 \pm 9 \mathrm{mag}$, blue- and redshifted lobe, respectively; see Methods) is smaller than that towards the HMYSO itself. The walls of the outflow cavities are acting as a mirror, scattering the light from the outbursting young star and allowing us to peer directly into the central accretion region. The right-hand panel of Fig. 2 compares our NIFS/Gemini spectrum (in black) of the redshifted outflow lobe with the SINFONI/VLT pre-outburst spectrum $^{14}$ of the same region (red spectrum). The new spectrum shows an increase in luminosity for both the continuum and lines $\left(\mathrm{H}_{2}, \mathrm{Br} \gamma\right)$, as well as the appearance of new emission lines, namely $\mathrm{CO}$ band-heads, Na I, He I, which are typically observed in young eruptive low-mass stars (EXors, FUors and MNors ${ }^{1,19-23}$ ) and are the typical signature of accretion disks, accretion and ejection activity.

Young eruptive low-mass stars $\left(\mathrm{M}_{*} \lesssim 2 \mathrm{M}_{\odot}\right)$ of these groups produce accretion bursts lasting from a few weeks up to decades, and with accretion luminosities up to thousands of solar luminosities ${ }^{1}$. During the burst, the mass-accretion rate $\left(\dot{M}_{\text {acc }}\right)$ usually increases from one (EXors) to several (MNors, FUors) orders of magnitude with respect to quiescence ${ }^{1,23}$. CO band-heads and $\mathrm{NaI}$ lines originate from the outer layer of the disk (within 1 a.u. from the central source in low-mass YSOs). The inner disk atmosphere is heated up to temperatures of a few thousand kelvin (2,000-4,000 K) by the accretion burst ${ }^{1}$ and these lines show up in emission. In 

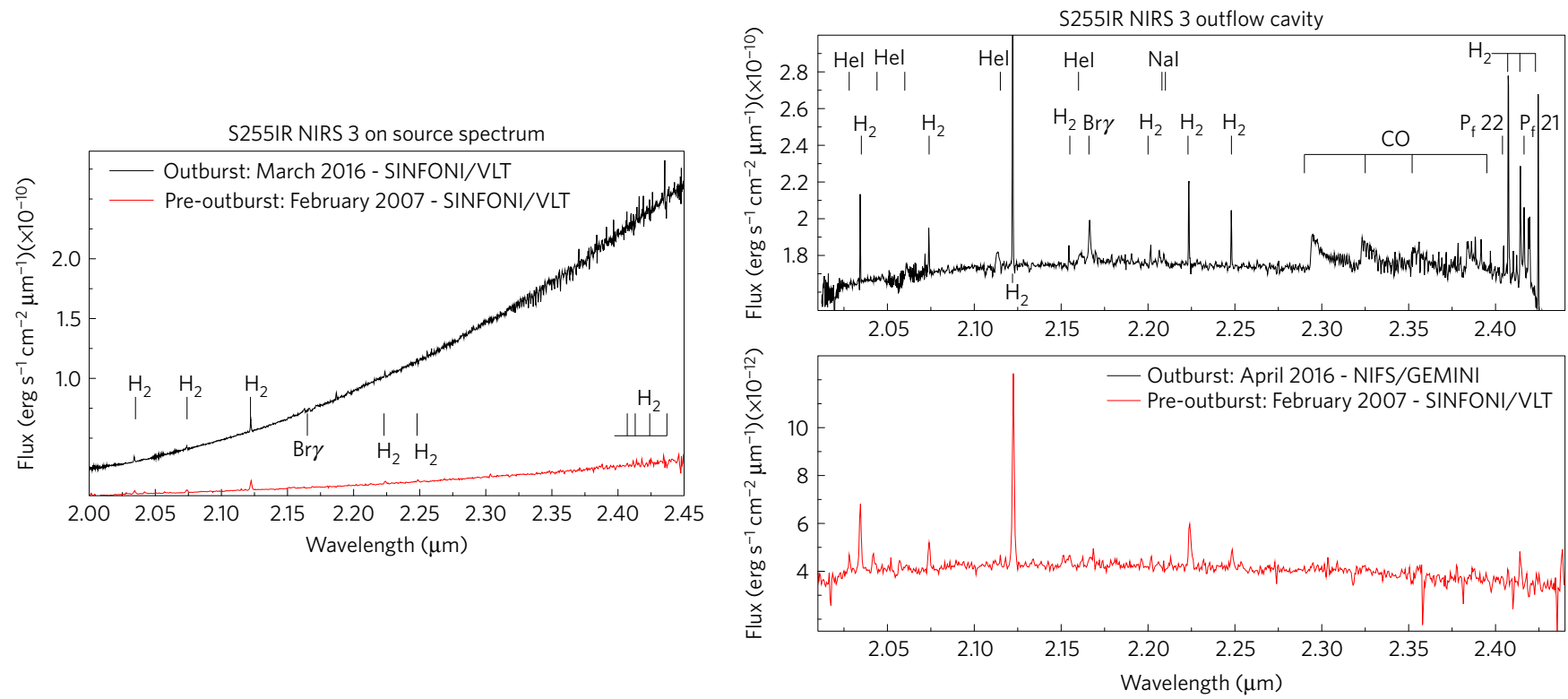

Figure 2 | Pre- and outburst K-band spectra of S255IR NIRS 3 (left) and its redshifted outflow cavity (right). Left: SINFONI/VLT pre-outburst (in red) and outburst (in black) K-band spectra of S255IR NIRS 3. Right: SINFONI/VLT pre-outburst (in red) and outburst (in black) K-band spectra of the redshifted outflow cavity of S255IR NIRS 3. The spectrum in the outburst phase shows a large number of emission lines typical of disk-mediated accretion outbursts.

contrast, both $\mathrm{He} \mathrm{I}$ and $\mathrm{Br} \gamma$ lines are emitted closer to the central source ( $\lesssim 0.1$ a.u.) and may originate from accretion onto the star and/or from disk winds ${ }^{20}$.

The total luminosities of the $\mathrm{Br} \gamma\left(2.2 \mathrm{~L}_{\odot}\right)$, $\mathrm{He} \mathrm{I}\left(1.2 \mathrm{~L}_{\odot}\right)$, $\mathrm{NaI}$ $\left(0.7 \mathrm{~L}_{\odot}\right)$ and $\mathrm{CO}_{v=2-0}\left(22 \mathrm{~L}_{\odot}\right)$ lines during the burst of NIRS 3 are from three to four orders of magnitude larger than those observed in EXors and MNors. Drawing a parallel between high-mass and low-mass YSOs, this evidence suggests that the size of the disk emitting region as well as the energy released by the burst are much larger in the present case. Indeed, the luminosity derived from the spectral energy distribution (SED) of NIRS 3 (see Fig. 3) grows from $\left(2.9 \pm_{0.7}^{1}\right) \times 10^{4} \mathrm{~L}_{\odot}$ (blue data points) to $\left(1.6 \pm_{0.3}^{0.4}\right) \times 10^{5} \mathrm{~L}_{\odot}($ red data points) during the burst (PANIC, GROND, VLT/SINFONI, SOFIA/FORCAST and FIFI-LS data), corresponding to an increase in accretion luminosity $\left(\Delta L_{\text {acc }}\right)$ of $\left(1.3 \pm_{0.3}^{0.4}\right) \times 10^{5} \mathrm{~L}_{\odot}$ and an energy release of $(1.2 \pm 0.4) \times 10^{46} \mathrm{erg}$ from the beginning of the burst until mid-April 2016 ( 9 months, according to our latest observations), when the source was still in burst. This latter amounts to an accreted mass of about two Jupiter masses (namely $\sim 3.4 \times 10^{-3} \mathrm{M}_{\odot}$, see Methods). The derived quantities are about four orders of magnitude larger than what is found in EXors and MNors, making this the most luminous accretion burst ever detected in a YSO. Moreover, assuming that the mass of the central source is $\sim 20 \mathrm{M}_{\odot}$ and its radius is equal to $10 \mathrm{R}_{\odot}$ (approximately the radius of a $\sim 20 \mathrm{M}_{\odot}$ star on the zero-age main sequence), from $\Delta L_{\text {acc }}$ we infer that $\dot{M}_{\text {acc }}$ is boosted to $(5 \pm 2) \times 10^{-3} \mathrm{M}_{\odot} \mathrm{yr}^{-1}$ (see Methods). The inferred value is probably a lower limit, as the radius of a massive protostar should be several times larger than that of a main sequence $\operatorname{star}^{24,25}$. Nevertheless, the inferred mass-accretion rate of this HMYSO burst is at least three orders of magnitude higher than those of EXors and MNors.

The accretion burst discovered in S255IR NIRS 3 adds fundamental information to our understanding of the high-mass star formation process. Our observations finally confirm that HMYSOs form through accretion disks at high mass-accretion rates. Moreover, they also provide an observational proof of episodic accretion, possibly originating from disk fragmentation. Here, the timescale and energetics of the outburst are more consistent with disk fragmentation rather than stellar merger ${ }^{26}$ (see Methods).

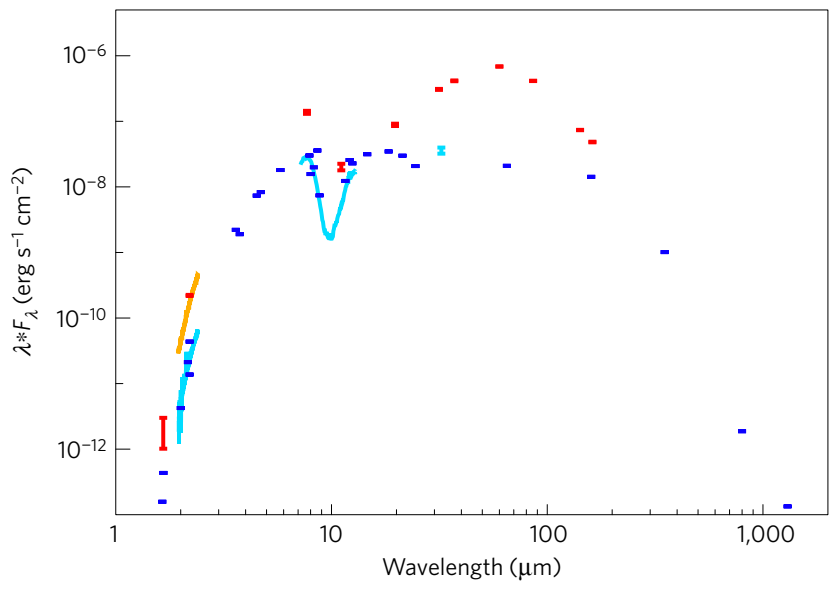

Figure 3 | Pre- (cyan and blue) and outburst (orange and red) spectral energy distributions (SEDs) of S255IR NIRS 3. Dark colours indicate photometric measurements, while light colours denote spectra.

In this respect, high-mass star formation can be considered as a scaled up version of the process by which low-mass stars are born. The main differences are that massive stars would form through larger accretion disks with much higher mass-accretion rates $\left(\geq 10^{-4} \mathrm{M}_{\odot} \mathrm{yr}^{-1}\right)$, and on shorter timescales.

High mass-accretion rates and the presence of an accretion disk are fundamental ingredients to circumvent the intense radiation pressure of the massive star, which otherwise might reduce, and even halt accretion. They allow further accretion to proceed even after the hydrogen burning starts ${ }^{7}$. At variance with low-mass protostars, the timescale for gravitational contraction (Kelvin-Helmholtz time) is shorter than the timescale for accretion in HMYSOs, producing a strong radiation field ${ }^{25}$. The circumstellar disk reduces the radiation pressure, allowing most of the radiation to escape through the bipolar cavities ${ }^{27}$. Indeed the light echo and the increase in brightness of the outflow cavities in NIRS 3 confirm this picture.

Finally, as with low-mass protostars, the accretion process would not be continuous but episodic. This would also explain the 
observation of several knots in jets from HMYSOs ${ }^{28}$, assuming major outflows events can be linked to major accretion events. Indeed, the morphology of different gas tracers along the outflow axis of NIRS 3, which shows a discrete number of knots, suggests that the source experienced multiple bursts within the past few thousand years ${ }^{13-15}$. Our burst detection proves the erratic behaviour of the accretion process in HMYSOs. Indeed, several radiation hydrodynamic simulations predict the onset of accretion variability in high-mass star formation ${ }^{6,7,11}$. Notably, episodic accretion might also play an important role in regulating the ionizing radiation, bloating the central source, and prolonging the accretion time during the Ultra-Compact H II (UCH II) phase ${ }^{29}$.

\section{Methods}

Methods, including statements of data availability and any associated accession codes and references, are available in the online version of this paper.

Received 12 July 2016; accepted 29 September 2016; published online 14 November 2016

\section{References}

1. Audard, M. et al. Episodic accretion in young stars. in Protostars and Planets VI (eds Beuther, H., Klessen, R., Dullemond, C. P. \& Henning, T.) 387-410 (Univ. Arizona Press, 2014).

2. Kratter, K. M. \& Matzner, C. D. Fragmentation of massive protostellar discs. Mon. Not. R. Astron. Soc. 373, 1563-1576 (2006).

3. Krumholz, M. R., Klein, R. I. \& McKee, C. F. Radiation-hydrodynamic simulations of collapse and fragmentation in massive protostellar cores. Astrophys. J. 656, 959-979 (2007).

4. Vorobyov, E. I. \& Basu, S. Variable protostellar accretion with episodic bursts. Astrophys. J. 805, A115 (2015).

5. Tan, J. C. et al. Massive star formation. in Protostars and Planets VI (eds Beuther, H., Klessen, R., Dullemond, C. P. \& Henning, T.) 149-172 (Univ. Arizona Press, 2014).

6. Kuiper, R., Klahr, H., Beuther, H. \& Henning, T. Three-dimensional simulation of massive star formation in the disk accretion scenario. Astrophys. J. 732, 1-11 (2011).

7. Klassen, M., Pudritz, R. E., Kuiper, R., Peters, T. \& Benerjee, R. Simulating the formation of massive protostars. I. Radiative feedback and accretion disks. Astrophys. J. 823, A28 (2016).

8. Kraus, S. et al. A hot compact dust disk around a massive young stellar object. Nature 466, 339-342 (2010).

9. Caratti o Garatti, A. et al. Tracing jet emission at the base of a high-mass YSO. First AMBER/VLTI observations of the $\mathrm{Br} \gamma$ emission in IRAS 13481-6124. Astron. Astrophys. 589, L4 (2016).

10. Tan, J. C. \& McKee, C. F. The formation of the first stars. I. Mass infall rates, accretion disk structure, and protostellar evolution. Astrophys. J. 603, 383-400 (2004)

11. Krumholz, M. R., Klein, R. I., McKee, C. F., Offner, S. S. R. \& Cunningham, A. J. The formation of massive star systems by accretion. Science 323, 754-757 (2009).

12. Stecklum, B. et al. The methanol maser flare of S255IR and an outburst from the high-mass YSO S255IR-NIRS3-more than a coincidence? The Astronomer's Telegram 8732, 1 (2016).

13. Zinchenko, I. et al. The disk-outflow system in the S255IR area of high-mass star formation. Astrophys. J. 810, A10 (2015).

14. Wang, Y. et al. Different evolutionary stages in the massive star-forming region S255 complex. Astron. Astrophys. 527, A32 (2011).
15. Burns, R. A., Handa, T., Nagayama, T., Sunada, K. \& Omodaka, T. $\mathrm{H}_{2} \mathrm{O}$ masers in a jet-driven bowshock: episodic ejection from a massive young stellar object. Mon. Not. R. Astron. Soc. 460, 283-290 (2016).

16. Boley, P. A. et al. The VLTI/MIDI survey of massive young stellar objects. Sounding the inner regions around intermediate- and high-mass young stars using mid-infrared interferometry. Astron. Astrophys. 558, A24 (2013).

17. Fujisawa, K. et al. A flare of methanol maser in S255. The Astronomer's Telegram 8286, 1 (2015).

18. Sobolev, A. M., Cragg, A. M. \& Goodfrey, P. D. Pumping of Class II methanol masers. II. The $5_{1}-6_{0} A^{+}$transition. Astron. Astrophys. 433, 211-220 (1997)

19. Lorenzetti, D. et al. Near-infrared spectroscopic monitoring of EXor variables: first results. Astrophys. J. 693, 1056-1073 (2009).

20. Kóspál, Á. et al. Near-infrared spectroscopy of EX Lupi in outburst. Astrophys. J. 736, A72 (2011)

21. Caratti o Garatti, A. et al. LBT/LUCIFER near-infrared spectroscopy of PV Cephei. An outbursting young stellar object with an asymmetric jet. Astron. Astrophys. 693, A66 (2013).

22. Reipurth, B. \& Aspin, C. Infrared spectroscopy of Herbig-Haro energy sources. Astron. J. 114, 2700-2707 (1997)

23. Contreras Peña, C. et al. Infrared spectroscopy of eruptive variable protostars from VVV. Preprint at http://arxiv.org/abs/1602.06269 (2016).

24. Hosokawa, T. \& Omukai, K. Evolution of massive protostars with high accretion rates. Astrophys. J. 691, 823-846 (2009).

25. Hosokawa, T., Yorke, H. W. \& Omukai, K. Evolution of massive protostars via disk accretion. Astrophys. J. 721, 478-492 (2010).

26. Bally, J. \& Zinnecker, H. The birth of high-mass stars: accretion and/or mergers? Astron. J. 129, 2281-2293 (2005)

27. Krumholz, M. R., McKee, C. F. \& Klein, R. I. How protostellar outflows help massive stars form. Astrophys. J. Lett. 618, 757-768 (2005).

28. Caratti o Garatti, A. et al. A near-infrared spectroscopic survey of massive jets towards extended green objects. Astron. Astrophys. 573, A82 (2015).

29. Hosokawa, T. et al. Formation of massive primordial stars: intermittent UV feedback with episodic mass accretion. Astrophys. J. 824, A119 (2016).

\section{Acknowledgements}

A.C.o.G., R.G.L. and T.P.R. were supported by Science Foundation Ireland, grant 13/ERC/I2907. A.S. was supported by the Deutsche Forschungsgemeinschaft (DFG) Priority Program 1573. We thank the ESO Paranal and Gemini Observatory staff for their support. B.S. thanks Sylvio Klose for helpful discussions concerning the light echo. This research is partly based on observations collected at the VLT (ESO Paranal, Chile) with programme 296.C-5037(A) and at the Gemini Observatory (Program ID GN-2016A-DD-5). Gemini Observatory is operated by the Association of Universities for Research in Astronomy, under a cooperative agreement with the NSF on behalf of the Gemini partnership: the National Science Foundation (United States), the National Research Council (Canada), CONICYT (Chile), Ministerio de Ciencia, Tecnología e Innovación Productiva (Argentina), and Ministério da Ciência, Tecnologia e Inovação (Brazil)

\section{Author contributions}

A.C.o.G., B.S. and R.G.L. wrote the initial manuscript and worked on the data reduction and analysis. R.G.L. supported SINFONI observations. A.C.o.G., B.S. and J.E. are the principal investigators of the ESO, Calar Alto, the Gemini, and the SOFIA proposals, respectively. A.S., R.C. and L.M. worked on the maser and radio data. T.P.R., A.S., R.C., L.M., C.M.W., R.D.O. and W.J.d.W. are coauthors of the proposals. J.G. provided GROND data. A.K., C.F. and R.K. supported SOFIA observations. J.M.I. supported PANIC observations. All coauthors commented on the manuscript.

\section{Additional information}

Reprints and permissions information is available online at www.nature.com/reprints. Correspondence and requests for materials should be addressed to A.C.o.G.

\section{Competing financial interests}

The authors declare no competing financial interests. 


\section{Methods}

Infrared imaging of the burst. Near-infrared imaging at various epochs was performed with PANIC ${ }^{30}$ at the Calar Alto 2.2-m telescope and the Gamma-Ray Burst Optical/Near-Infrared Detector (GROND) ${ }^{31}$ at the La Silla 2.2-m telescope. Basic image processing was performed by the instrument teams using the corresponding data pipelines. The photometric calibration was done using the Two Micron All Sky Survey (2MASS) catalogue ${ }^{32}$. Although short detector integration times were applied for the Ks band, partial saturation of the bright target and field stars of similar brightness was unavoidable, in particular under good seeing conditions (our typical seeing was $\lesssim 1^{\prime \prime}$ ). This was accounted for by a continuous extension of the linear fit between catalogue and instrumental magnitudes with a parabola for the brightest objects. Flux densities for NIRS 3 were generally derived using the APER procedure from the IDL Astronomy Library ${ }^{33}$, taking the local background into account. Mid- and far-infrared flux densities were obtained by performing target-of-opportunity observations with FORCAST ${ }^{34}$ and FIFI-LS $S^{35,36}$ aboard SOFIA (principal investigator J. Eislöffel, proposal ID 04_0047). FORCAST images were taken using narrow-band filters centred at 7.7, 11.1, 19.7, 31.5 and $37.1 \mu \mathrm{m}$. The spectral windows for FIFI-LS were chosen to match the central wavelengths of the far-infrared AKARI filters-that is, 60, 90, 140 and $160 \mu \mathrm{m}$. The FIFI-LS spectral data cubes, calibrated by a FIFI-LS team member (C. F.), were collapsed and photometry was performed on the mean image.

The photometric values of the burst shown in Fig. 3, along with their error, photometric aperture, instrument and observation date, are reported in Table 1.

Light echo. To cancel the influence of non-uniform extinction for the assessment of the change of the scattered light distribution due to the burst, and to compensate the decreasing surface brightness with growing distance from the source, a ratio image between PANIC Ks and UKIDSS K frames was calculated. Before doing so, the PSFs of the K frame was convolved with a proper kernel to match that of the Ks frame. The applied photometric scaling factor was derived from the corresponding zero points of the images. This turned out to be correct, since the brightness ratio for field stars is of the order of unity. The resulting distribution has an asymmetric bipolar morphology. The asymmetry results from the inclination of the scattering cavities relative to the sky plane, leading to larger light distances for the blueshifted lobe for a given propagation period and vice versa.

The surfaces of scattered light of fixed travel time can be approximated as paraboloids with the star at the origin. Thereby, it can be shown that, at the onset of an outburst $(t=0)$, the size ratio between the back- and forward-scattering lobes is zero and increases to unity over time. Thus, an approximately equal extent of the scattering lobes of a YSO seen close to edge-on is expected only for steady-state illumination. Moreover, for the purpose of judging the lobe sizes, it must also be taken into account that forward scattering dominates in the blueshifted lobe, whereas backward scattering is prevalent in the redshifted lobe. Because of the different scattering efficiencies, the redshifted lobe will be less bright in general, and thus appear smaller for a given surface brightness sensitivity.

The same analysis on a later PANIC Ks image (February 2016) confirms the light echo by verifying both its propagation and dilution. For deriving the onset of the burst (mid-June 2015) we estimated the light travel time derived from the mean of the extent of both lobes.

Spectral energy distribution. As the energy released by the burst is thermalized by dust grains and radiated away in the infrared, pre-burst fluxes in this wavelength range are crucial for deriving the increase in luminosity. For this purpose, pre-outburst non-saturated IRAC images of S255IR (taken in sub-array mode, courtesy of G. Fazio, program ID 40440) were retrieved from the IPAC infrared science archive. Image mosaics were obtained from the dithered images for each channel using a custom IDL (IDL is a trademark of Exelis Visual Information Solutions) procedure. Flux densities for NIRS 3 were estimated as described above. Similarly, flux densities for the N60 and N160 AKARI bands were derived from the corresponding images after retrieval from the ISAS/JAXA archive (the wide AKARI channels centred at 90 and $140 \mu \mathrm{m}$ are saturated). These data were complemented with an archival ISO/SWS spectrum (courtesy D. Whittet), $H$ and Ks VLT/ISAAC photometry (private communication by S. Correia, ESO proposal ID 074.C-0772(B)) as well as flux densities from the literature ${ }^{13,14,37-39}$ and surveys (AKARI, BGPS, MSX, UKIDSS). The outburst SED was obtained using data from PANIC, GROND, SINFONI, FORCAST and FIFI-LS taken in February 2016. The pre- and burst luminosities were derived by integrating the dereddened SEDs and assuming a distance of $1.8 \pm 0.1 \mathrm{kpc}$. To deredden the SED we adopt our visual extinction $A_{V}=44 \pm 16 \mathrm{mag}$ and $R_{V}=3.1$ extinction law ${ }^{40}$. The resulting pre- and outburst luminosities are $\left(2.9 \pm_{0.7}^{1}\right) \times 10^{4} \mathrm{~L}_{\odot}$ and $\left(1.6 \pm_{0.3}^{0.4}\right) \times 10^{5} \mathrm{~L}_{\odot}$, respectively. The uncertainties were inferred from the small distance error and the uncertainty on the visual extinction. We also note that because of the close to edge-on view of its circumstellar disk, the estimated luminosity might represent a lower limit. The proper value may be up to two times higher ${ }^{41}$.
Table 1 | Flux densities of SED burst values.

\begin{tabular}{llllll} 
Wavelength & $\begin{array}{l}\text { Flux } \\
\text { density } \\
(\mu \mathrm{m})\end{array}$ & $\begin{array}{l}\text { Error } \\
(\mathrm{Jy})\end{array}$ & $\begin{array}{l}\text { Aperture } \\
\text { radius } \\
\left({ }^{\prime \prime}\right)\end{array}$ & & Instrument Date \\
\hline 1.66 & 0.0011 & 0.0005 & 0.6 & PANIC & $15 / 01 / 2016$ \\
2.2 & 0.1620 & 0.0032 & 0.6 & GROND & $18 / 02 / 2016$ \\
7.7 & 352.8 & 18.8 & 5 & FORCAST & $04 / 02 / 2016$ \\
11.1 & 74.8 & 8.6 & 6 & FORCAST & $04 / 02 / 2016$ \\
19.7 & 580.9 & 24.1 & 8 & FORCAST & $04 / 02 / 2016$ \\
31.5 & 3,223 & 56.8 & 10 & FORCAST & $04 / 02 / 2016$ \\
37.1 & 5,136 & 71.7 & 11 & FORCAST & $04 / 02 / 2016$ \\
60 & 13,720 & 97.2 & 10 & FIFI-LS & $01 / 03 / 2016$ \\
86 & 11,880 & 81.3 & 12 & FIFI-LS & $01 / 03 / 2016$ \\
142 & 3,490 & 24.2 & 20 & FIFI-LS & $01 / 03 / 2016$ \\
162 & 2,630 & 29.0 & 22 & FIFI-LS & $01 / 03 / 2016$
\end{tabular}

Infrared integral field unit spectroscopy. Our K-band $(1.95-2.5 \mu \mathrm{m})$ integral field unit (IFU) spectroscopic data of S255IR NIRS 3 consist of three data sets taken with SINFONI ${ }^{42}$ on VLT (ESO, Chile) with $\mathrm{R} \sim 4,000$ and $\mathrm{NIFS}^{43}$ on the Gemini North telescope with $\mathrm{R} \sim 5,300$. Adaptive-optics-assisted mode was used for all runs. The first SINFONI data set (26 February 2016) was centred on NIRS 3

(25 milliarcseconds (mas) pixel scale and field of view - FoV - of $\left.0.8^{\prime \prime} \times 0.8^{\prime \prime}\right)$. The second SINFONI data set (9 March 2016) was taken with the lowest spatial sampling (250 mas pixel scale and FoV of $\left.8^{\prime \prime} \times 8^{\prime \prime}\right)$ and maps an area of $\sim 11^{\prime \prime} \times 11^{\prime \prime}$ around NIRS 3 , covering NIRS 3 , NIRS 1 and their outflow cavities. NIFS data (100 mas pixel scale and FoV of $\left.3^{\prime \prime} \times 3^{\prime \prime}\right)$ were collected on the 8 April 2016 and map the redshifted outflow cavity covering an area of $\sim 6^{\prime \prime} \times 6^{\prime \prime}$.

SINFONI data were reduced with the standard reduction pipeline in GASGANO $^{44}$, which includes dark and bad pixel removal, flat-field and optical distortion correction, wavelength calibration with arc lamps, and image combination to obtain the final 3D data cube. NIFS data reduction was accomplished in a similar fashion using the Gemini package in IRAF.

All data were corrected for atmospheric transmission and flux calibrated by means of standard stars.

SINFONI pre-outburst IFU spectra, taken between February and March 2007, were retrieved from the ESO Data Archive and have already been published in a previous paper ${ }^{14}$. They map an area $\left(70^{\prime \prime} \times 70^{\prime \prime}\right)$ larger than our observations. To compare pre- and outburst data, spectra were extracted from our data cubes within an area of $1.5^{\prime \prime} \times 1.5^{\prime \prime}$ (centred on NIRS 3 source;

$\left.\operatorname{RA}(J 2000): 6^{\mathrm{h}} 12^{\mathrm{m}} 54.0^{\mathrm{s}} ; \operatorname{DEC}(J 2000):+17^{\circ} 59^{\prime} 23.1^{\prime \prime}\right)$ and $6^{\prime \prime} \times 6^{\prime \prime}$ (centred on RA(J200): $\left.6^{\mathrm{h}} 12^{\mathrm{m}} 54.4^{\mathrm{s}} ; \mathrm{DEC}(\mathrm{J} 2000):+17^{\circ} 59^{\prime} 24.7^{\prime \prime}\right)$ for NIRS 3 (Fig. 2, left panel) and the redshifted outflow cavity (Fig. 2, right panel), respectively.

Visual extinction variability versus accretion burst. In principle, large variations of the extinction towards NIRS 3 could be a possible cause of the infrared variability of NIRS 3 . However, this argument does not fit our observations for the following reasons.

First, the increase in luminosity is detected at NIR, MIR and FIR wavelengths. This implies that the variation in luminosity cannot be due to a change in visual extinction, which would indeed affect the NIR part of the SED but would just marginally affect the MIR part of the spectrum and would not affect its FIR portion. Second, the increase in luminosity at infrared wavelengths temporally matches the flares of the methanol masers in the radio. Moreover the maser positions match that of NIRS 3 (Sanna et al., in preparation). Third, in addition, the light echo observed at NIR wavelengths matches the timing of the $\mathrm{CH}_{3} \mathrm{OH}$ maser flares. Fourth, the increase in the SED luminosity matches the appearance $(\mathrm{CO}$, $\mathrm{He}$ I, Na I, lines) and increase in luminosity $\left(\mathrm{Br} \gamma, \mathrm{H}_{2}\right)$ of the infrared lines. Fifth, visual extinction affects the intensity of both lines and continuum as well as the continuum's colour. As the extinction affects both lines and continuum to the same extent, the equivalent width (EW) of the lines should not change. On the other hand, EWs and fluxes of $\mathrm{Br} \gamma$ and $\mathrm{H}_{2}$ lines, already present in the pre-outburst spectrum in the outflow cavity, show a large variability and are anti-correlated, as expected in accretion events ${ }^{45}$. This cannot be explained with extinction variability. Moreover, the slope of the K-band spectra on source and outflow cavities does not show a significant change before and during the outburst-that is, we do not detect any blueing of the spectra in 2016. Finally, as reported in the next subsection, the visual extinction towards the outflow cavities does not change significantly.

Therefore we infer that the visual extinction did not change significantly between 2007 and 2016 . 
Visual extinction towards the outflow cavities and on-source. To estimate the visual extinction towards both blue and redshifted lobes, we use pairs of lines from [FeII] $(2.016 / 2.254 \mu \mathrm{m})$ and $\mathrm{H}_{2}(2.034 / 2.437 \mu \mathrm{m}, 2.122 / 2.424 \mu \mathrm{m}, 2.223 / 2.413 \mu \mathrm{m})$ species that originate from the same upper level. We detect shocked emission lines ( $[\mathrm{FeII}]$ and $\mathrm{H}_{2}$ ) in two knots positioned in the blue- and redshifted lobes, respectively. Assuming that the emission arises from optically thin gas, the observed line ratios depend only on the differential extinction. The theoretical values are derived from the Einstein coefficients ${ }^{46}$ and frequencies of the transitions. We adopt the Rieke \& Lebofsky ${ }^{47}$ extinction law to correct for the differential extinction and compute $\mathrm{A}_{V}$. Values inferred are $\mathrm{A}_{V}=18 \pm 5 \mathrm{mag}$ $\left(\mathrm{A}_{V}([\mathrm{FeII}])=16 \pm 10 \mathrm{mag}\right.$ and $\left.\mathrm{A}_{V}\left(\mathrm{H}_{2}\right)=19 \pm 5 \mathrm{mag}\right)$ for the blueshifted lobe and $\mathrm{A}_{V}=28 \pm 9 \mathrm{mag}\left(\mathrm{A}_{V}([\mathrm{FeII}])=27 \pm 14 \mathrm{mag}\right.$ and $\left.\mathrm{A}_{V}\left(\mathrm{H}_{2}\right)=29 \pm 12 \mathrm{mag}\right)$ for the redshifted lobe. Similar values, but with larger uncertainties, are inferred from the pre-outburst spectra (2007) of the blueshifted $\left(\mathrm{A}_{V}\left(\mathrm{H}_{2}\right)=18 \pm 7 \mathrm{mag}\right)$ and redshifted $\left(\mathrm{A}_{V}\left(\mathrm{H}_{2}\right)=27 \pm 15 \mathrm{mag}\right)$ outflow cavities. These latter measurements suggest that the visual extinction towards the lobes did not change significantly.

We also infer the visual extinction towards NIRS 3 from the $\mathrm{H}_{2}$ lines detected in the outburst spectrum (Fig. 2, left panel), obtaining $\mathrm{A}_{V}\left(\mathrm{H}_{2}\right)=44 \pm 16$ mag. The inferred value is consistent with $\mathrm{A}_{V}=46 \mathrm{mag}$ from Simpson and colleagues ${ }^{39}$. Finally, from the pre-outburst $J-H$ and $H-K$ colours of the UKIDSS photometry, we obtain $\mathrm{A}_{V} \sim 48-62 \mathrm{mag}$ by assuming that NIRS 3 is a O6 spectral type positioned on the ZAMS. This latter is consistent with our previous estimate. Therefore we adopt $A_{V}\left(H_{2}\right)=44 \pm 16$ mag towards the source and use this value to deredden the SED.

Line luminosity. The line luminosities in the redshifted lobe were inferred from the dereddened line fluxes using $\mathrm{A}_{V}=28 \pm 9 \mathrm{mag}$ and assuming a distance to the object of $1.8 \pm 0.1 \mathrm{kpc}^{15}$.

Energy of burst, accreted mass and mass-accretion rate. The burst energy ( $E=\Delta L_{\text {acc }} \times \Delta t$, where $\Delta t$ is the length of the burst) delivered so far (until mid-April 2016, the date of the last available observation) by the burst is inferred from $\Delta L_{\text {acc }}=\left(1.3 \pm_{03}^{0.4}\right) \times 10^{5} \mathrm{~L}_{\odot}$, obtained from the pre- and outburst SED, and considering that the burst began around mid-June 2015. The accreted mass is inferred assuming that the stellar radius is $R_{*}=10 \mathrm{R}_{\odot}$ and using $E=G M_{*} M_{\text {acc }} / R_{*}$, where $G$ is the gravitational constant, $M_{*}$ is the mass of the star and $M_{\text {acc }}$ is the accreted mass. Finally, the mass-accretion rate is obtained from $\dot{M}_{\text {acc }} \sim\left(2 \Delta L_{\text {acc }} R_{*}\right) /\left(G M_{*}\right)$.

Disk-accretion by fragmentation versus merging. A conceptual question involves whether our observations can rule out the possibility that what we are seeing is not disk fragmentation but stellar capture and merger via tidal disruption ${ }^{26}$. This scenario proposes that massive stars build up by capturing other stars in disks, then tidally disrupting them. However, both timescales and energetics of the outburst of S255 NIRS 3 seem to be inconsistent with such a scenario. For example, assuming that the mass of the central object is $\sim 20 \mathrm{M}_{\odot}$, the merger with a brown dwarf of $0.1 \mathrm{M}_{\odot}$ would produce an energy of $\sim 5 \times 10^{47} \mathrm{erg}$ released in $\sim 10^{4} \mathrm{yr}$. These values are much larger than what we inferred from the outburst of NIRS 3.

Data availability. The data sets generated and analysed during the current study are not publicly available due to a proprietary period restriction of 12 months. After this period ESO/SINFONI and GROND data will become publicly available from the European Southern Observatory science archive (http://archive.eso.org/eso/eso_archive_main.html) under programs ID 296.C-5037(A) and 096.A-9099(A); Gemini/NIFS data from the Gemini Observatory archive (https://archive.gemini.edu/searchform) under program ID GN-2016A-DD-5; SOFIA/FORCAST and FIFI-LS data from the SOFIA science archive (https://dcs.sofia.usra.edu/dataRetrieval/SearchScienceArchive InfoBasic.jsp) under program ID 04_0047. Upon request the authors will provide all data supporting this study.

\section{References}

30. Baumeister, H. et al. PANIC: the new panoramic NIR camera for Calar Alto. SPIE 7014, 70142R (2008).

31. Greiner, J. et al. GROND-a 7-channel imager. Pub. Astron. Soc. Pac. 120, 405-424 (2008).

32. Skrutskie, M. F. et al. The two micron all sky survey (2MASS). Astron. J. 131, 1163-1183 (2006).

33. Landsman, W. B. The IDL Astronomy User's Library. in Astronomical Data Analysis Software and Systems II (eds Hanisch, R. J., Brissenden, R. J. V. \& Barnes, J.) 246-248 (A.S.P. Conference Series 52, 1993).

34. Adams, J. D. et al. FORCAST: a first light facility instrument for SOFIA. SPIE $7735,77351 \mathrm{U}(2010)$.

35. Klein, R. et al. FIFI LS: the far-infrared integral field spectrometer for SOFIA. SPIE 6269, 62691F (2006).

36. Klein, R. et al. FIFI-LS: the facility far-infrared spectrometer for SOFIA. SPIE 9147, 8 (2014).

37. Longmore, S. N., Burton, M. G., Minier, V. \& Walsh, A. J. Mid-infrared source multiplicity within hot molecular cores traced by methanol masers. Mon. Not. R. Astron. Soc. 369, 1196-1200 (2006).

38. Itoh, Y. et al. Near-infrared observations of S 255-2: The heart of a massive YSO cluster. Pub. Astron. Soc. Japan 369, 495-500 (2001).

39. Simpson, J. P. et al. Hubble space telescope NICMOS polarization observations of three edge-on massive young stellar objects. Astrophys. J. 700, 1488-1501 (2009).

40. Draine, B. Scattering by interstellar dust grains. I. Optical and ultraviolet. Astrophys. J. 598, 1017-1025 (2003).

41. Whitney, B. A., Wood, K., Bjorkman, J. E. \& Wolff, M. J. Two-dimensional radiative transfer in protostellar envelopes. I. Effects of geometry on class I sources. Astrophys. J. 591, 1049-1063 (2003).

42. Eisenhauer, F. et al. SINFONI-Integral field spectroscopy at 50 milli-arcsecond resolution with the ESO VLT. SPIE 4841, 1548-1561 (2003).

43. McGregor, P. J. et al. Gemini near-infrared integral field spectrograph (NIFS). SPIE 4841, 1581-1591 (2003).

44. Modigliani, A. et al. The SINFONI pipeline ArXiv Astrophysics e-prints 0701297, 10 (2007).

45. Lorenzetti, D. et al. Interpreting the simultaneous variability of near-IR continuum and line emission in young stellar objects. AperS 343, 535-539 (2013).

46. Deb, N. C. \& Hibbert, A. log gf values for astrophysically important transitions Fe II. Astron. Astrophys. 561, A32 (2014).

47. Rieke, G. H. \& Lebofsky, M. J. The interstellar extinction law from 1 to 13 microns. Astrophys. J. 288, 618-621 (1985). 